\title{
Analysis of Control Valves Stiction Quantification Tool
}

\author{
M Gaberalla M K Elarafi ${ }^{1}$
}

H. Zabiri ${ }^{*}, 2$

${ }^{1}$ BARDEGG - 2 Project (Package 3) Projects \& Engineering Division (P\&E) - PCSB, Malaysia

2 Chemical Engineering Department, Universiti Teknologi Petronas (UTP) 32610 Bandar Seri Iskandar, Perak, Malaysia. Tel: +605-3687625 Fax: +605- 3656176

*e-mail: haslindazabiri@utp.edu.my

Control valve stiction is considered as one of the main sources of control loops nonlinearities which impacts plants profitability. In turn, this phenomenon hinders the plant from being operated at optimal conditions. Therefore, an efficient and accurate stiction quantification algorithm is required for accurate stiction compensation and timely scheduling of control valve maintenance. This research investigates the robustness and recommends improvements to the previously developed stiction quantification approach by Zabiri et al. The approach was tested under several operating conditions which were simulated in five case studies by using MATLAB software. The case studies investigated the impact of a wide range of stiction values, controller tuning, disturbance, time delay and noise on the quantification approach. The algorithm was found to be robust since it quantified the correct values of stiction regardless of the operating conditions. It was found that the accuracy of the quantification results depends on the process model accuracy, number of data samples and the search resolution. A number of improvements were recommended and validated by simulation in order to further enhance the current quantification approach. As conclusion, the algorithm can be applied on any type of process due to its robustness.

Keywords : Control valve stiction, quantification, NN, robustness test

\section{INTRODUCTION}

Control valve stiction and its deteriorating impacts on process plant performance is a broad research area. The topic itself is further split in the literature into subtopics such as stiction modeling, detection, quantification and compensation as classified in (AlSamarraie 2013, Brásio et al. 2014, Bedoui et al. 2014, Capaci et al. 2015).
Control valve stiction is one of the most commonly faced constraints that hinders the plant from being operated optimally. Therefore, great amount of work in the academic and industrial fields have been done in the past ten years as summarized in (Brásio et al. 2014, Capaci et al. 2015). In the literature, the area of stiction modeling and identification is considered mature, unlike stiction quantification which is still considered an open research area as 
mentioned in Zabiri et al. (2009) and Capaci et al. (2015).

Stiction detection mechanisms falls into four categories which are crosscorrelation, limit cycles patterns, nonlinearity detection and waveform shape-based (Capaci et al. 2015). Valve stiction quantification plays a vital role in stiction compensation and valve maintenance scheduling which ties back to product quality and profits. Stictioninduced oscillation has very unique MVOP data pattern, however, MV data that is the actual stem position is not available most of the time at the plant control system. Hence, stiction detection and quantitation mainly depend on the available data that is PV-OP. However, using PV-OP data poses a challenge because other disturbances-induced oscillation has similar patterns of PV-OP with stiction patterns which make it more difficult to differentiate (Capaci et al. 2015).

Brásio et al. (2014) and Capaci et al. (2015) conducted very extensive literature review on stiction in which they captured all the recent works on the topic until 2015. The summary of stiction quantification works is listed in Table 1 below. Capaci et al. (2015) found that Hammerstein system identification approach is the most common type of approach used for stiction quantification. The authors cited 27 techniques for stiction quantification of which $60 \%$ depends Hammestrein system identification approach. Zabiri et al. (2009), on the other hand, developed a stiction quantification method that depends on Artificial Neural Network process model and Choudhury's valve stiction model as shown in Capaci et al. (2015). This method has the advantage of being applicable on all process loops as mentioned by Brásio et al. (2014).

The scope of this paper focuses on control valve stiction quantification as a continuation to the research work done by Zabiri et al. (2009). The motive behind conducting this research is to contribute to finding a practical, efficient and accurate approach for stiction quantification. A contribution that can help in eliminating the deteriorating effects of stiction, lowering the plant maintenance cost due to the wear and tear of costly control valves and in turn increasing the plant profitability. Especially when considering the falling oil prices and the global recession that the oil and gas industry has undergone since 2014 . The objectives of this work are to test the robustness of the developed quantification method under several process condition scenarios, and to improve the developed quantification approach based on the results from the robustness test. This paper starts with the methodology in the next section, followed by results and discussion in Section 3, and finally, a conclusion is drawn and a recommendation is given.

\section{METHODOLOGY}

\section{Zabiri's Stiction Quantification Algorithm}

The works presented by Zabiri et al. (2009) utilizes artificial neural networks and Coudhury's stiction model with error minimization algorithm for stiction 
Table 1. Stiction quantification approaches (Capaci et al. 2015)

\begin{tabular}{|c|c|c|c|c|}
\hline \multirow{3}{*}{ Method } & \multicolumn{4}{|c|}{ Features } \\
\hline & \multirow[b]{2}{*}{ Type } & \multicolumn{2}{|c|}{ Blocks } & \multirow{2}{*}{$\begin{array}{c}\text { Application } \\
\text { on Industria } \\
\text { Data }\end{array}$} \\
\hline & & NL Model & $\begin{array}{c}\text { LIN } \\
\text { Model }\end{array}$ & \\
\hline Choudhury et al. & $P V(O P)$ fitting & - & - & $\sqrt{ }$ \\
\hline Cuadros et al. & $P V(O P)$ fitting & - & - & $x$ \\
\hline Yamashita & $P V(O P)$ fitting & - & - & $\sqrt{ }$ \\
\hline Stenman et al. & Hammerstein Id. & Stenman & ARX & $x$ \\
\hline Srinivasan et al. & Hammerstein Id. & Stenman & ARMAX & $\sqrt{ }$ \\
\hline Lee et al. & Hammerstein Id. & $\mathrm{He}$ & ARX & $\sqrt{ }$ \\
\hline Choudhury et al. & Hammerstein Id. & Choudhury & ARX & $\sqrt{ }$ \\
\hline Jelali [64] & Hammerstein Id. & Kano & ARMAX & $\sqrt{ }$ \\
\hline $\begin{array}{l}\text { Farenzena and } \\
\text { Trierweiler }\end{array}$ & Hammerstein Id. & Kano & ARMAX & $\sqrt{ }$ \\
\hline $\begin{array}{l}\text { Ivan and } \\
\text { Lakshminarayanan }\end{array}$ & Hammerstein Id. & He (modified) & ARMAX & $\sqrt{ }$ \\
\hline Karra and Karim & Hammerstein Id. & Kano & EARMAX & $\sqrt{ }$ \\
\hline $\begin{array}{l}\text { Sivagamasundari and } \\
\text { Sivakumar }\end{array}$ & Hammerstein Id. & $\mathrm{He}$ & $A R X$ & $\sqrt{ }$ (pilot) \\
\hline Shang et al. & Hammerstein Id. & Chen & ARX & $\sqrt{ }$ (pilot) \\
\hline Brasio et al. & Hammerstein Id. & Chen & ARX & $\mathrm{x}$ \\
\hline Srinivasan B. et al. & Hammerstein Id. & Stenman & ARMAX & $\sqrt{ }$ \\
\hline $\begin{array}{l}\text { Bacci di Capaci and } \\
\text { Scali }\end{array}$ & Hammerstein Id. & Kano & $A R X$ & $\sqrt{ }$ \\
\hline Bacci di Capaci et al. & Hammerstein Id. & Kano/He & 5 types & $\sqrt{ }$ (pilot) \\
\hline Wang and Zhang & Hammerstein Id. & Asymmetric & ARX & $\sqrt{ }$ \\
\hline Fang and Wang & Hammerstein Id. & Preisach & ARX & $\sqrt{ }$ \\
\hline Wang and Wang & Hamm - Wiener & Chen & Wiener & $x$ \\
\hline Romano and Garcia & Hamm - Wiener & Kano & Wiener & $\sqrt{ }$ \\
\hline $\begin{array}{l}\text { Ulaganathan and } \\
\text { Rengaswamy }\end{array}$ & Hamm - Wiener & Stenman & Wiener & $\sqrt{ }$ \\
\hline Chitralekha et al. & $\begin{array}{l}\text { Unknown input } \\
\text { observer }\end{array}$ & (Choudhury) & - & $\sqrt{ }$ \\
\hline Zabiri et al. & Neural Network & Choudhury & - & $x$ \\
\hline Nallasivam et al. & $\begin{array}{l}\text { Volterra Model - } \\
\text { Based }\end{array}$ & Stenman & Volterra & $\sqrt{ }$ \\
\hline Araujo et al. & $\begin{array}{l}\text { Describing Function } \\
\text { Semiphysical }\end{array}$ & DF & ARX & $\sqrt{ }$ \\
\hline He and Wang & Stiction Model & He (3 parameters) & - & $\sqrt{ }$ \\
\hline
\end{tabular}

quantification. The details are explained in the following subsections.

\section{Choudhury's Stiction Model and Plant}

Zabiri et al. quantification algorithm depends on Choudhury's stiction model shown in (Choudhury et al. 2005, 2006,
2008) for generating sticky manipulated variable (MV) data. The signal and data flow chart of Choudhury's stiction model is shown below in Fig.1. The flow chart can be summarized as follows:

1. The controller output is converted from 4-20 mA to travel \% by a look-up table. 


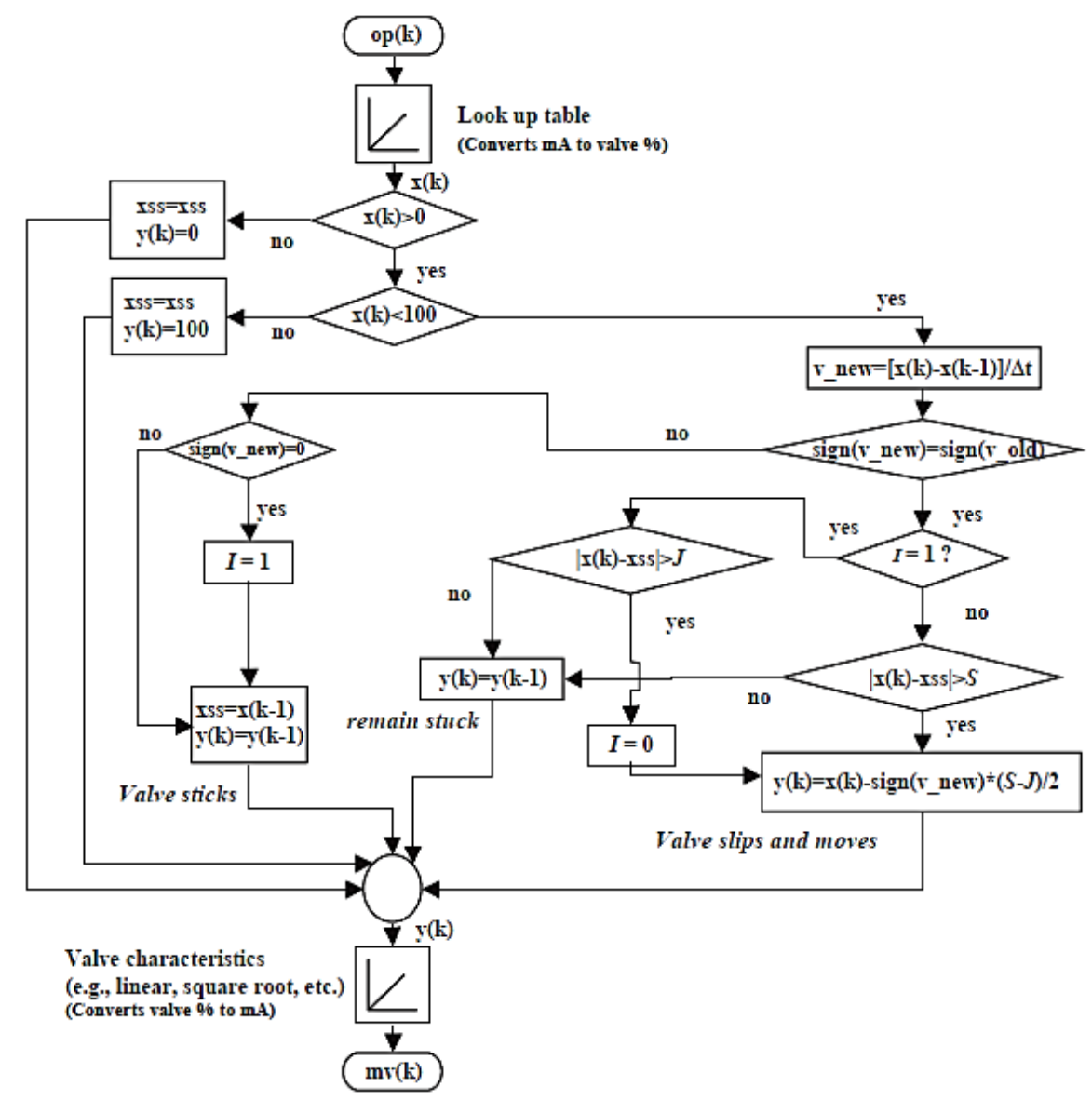

Fig.1: Signal and data flow chart of Choudhury's model (Brásio et al. 2014, Choudhury et al. 2008)]

2. The algorithm checks for saturation conditions whether the valve is fully closed or fully open.

3. If the valve is not saturated, then the algorithm calculates the slope of the controller output signal.

4. The direction of the slope is used to determine the beginning of the valve stiction as follows:

a. If there is a change in slope sign form + to - or vice versa, it indicates that the valve reversed its direction and the stiction starts. The controller output at the stiction point is recorded in Xss.

b. If there is a change in slope sign form + or - to zero, it indicates that the valve gets stuck in the moving phase. At this point the indicator (I) is set to $\mathrm{I}=1$ and the controller output at the stiction point is recorded in Xss.

c. If the difference value between the controller input and Xss is greater than $\mathrm{S}$ or J the valve slips and moves otherwise it remains stuck.

d. The output is calculated using (1) below whenever the valve slips and moves.

Output $=$ controller input $-\operatorname{sign}($ slope $) * \frac{(S-J)}{2}$

5. The output is converted to form travel $\%$ to 4-20 mA using a look-up table.

The plant that is used for sticky data generation is shown in Fig.1. It consists of 


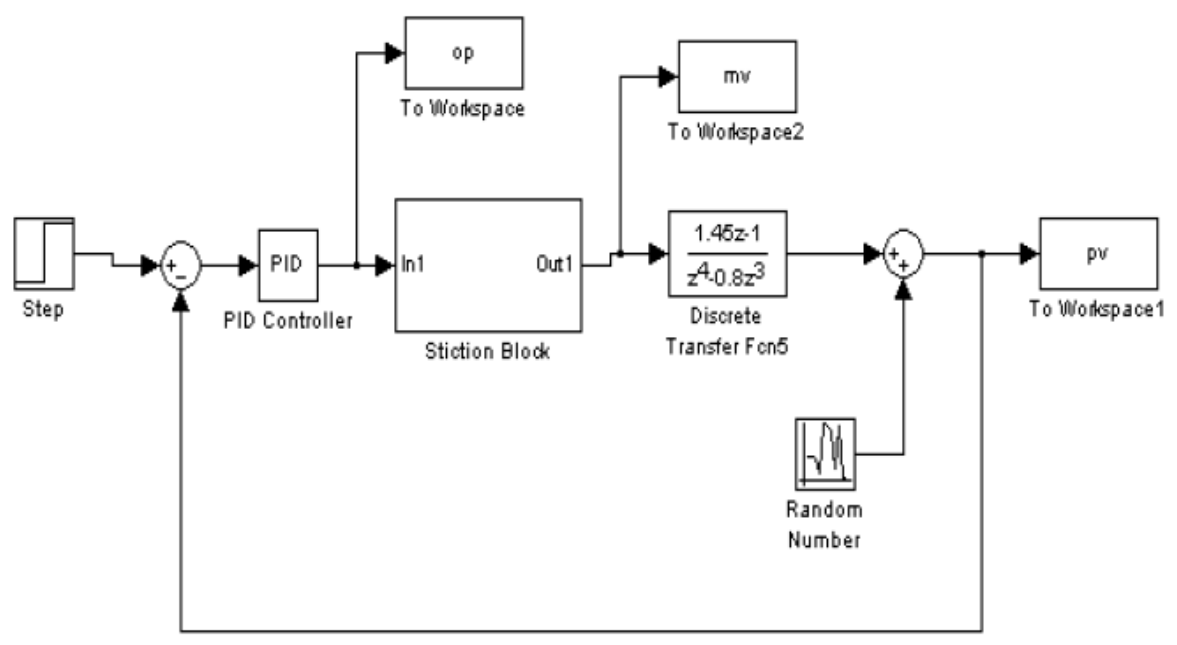

Fig.2: SIMULINK block diagram for stiction data generation

a linear first order transfer function as shown below. The loop is controlled by $\mathrm{PI}$ contoller that has the tuning settings shown in Table 2 (Control 2005, Zabiri et al. 2009).

$G(z)=\frac{1.45 z-1}{z^{3}-0.8 z^{2}}$

Table 2. PI Controller Parameters

\begin{tabular}{ccc}
\hline No. & $\boldsymbol{K}_{\boldsymbol{c}}$ & $\boldsymbol{T}_{\boldsymbol{i}}$ \\
\hline 1 & 0 & 0 \\
\hline 2 & 1 & 0.3 \\
\hline 3 & 5 & 1 \\
\hline
\end{tabular}

\section{Artificial Neural Network}

Artificial Neural Network (ANN) is a group of artificial neurons interconnected in a way that resembles the human brain neural network. It is an artificial intelligence mathematical approach that is wildly used in nonlinear data modeling. The network consists of input layer, hidden layers and output layer. Each layer consists of neurons which consist adjustable weights, bias, threshold and activation function. The neuron calculates a weighted summation of inputs and compares it to a threshold. If the summation is greater than the threshold, the output is set to a certain value based on a specific activation function as shown in Fig.3. The weights are dynamically adjusted based on mathematical algorithm in order to approximate the output of the network to the desired output. Therefore, ANN is categorized as an adaptive system as mentioned in Zabiri et al. (2009).

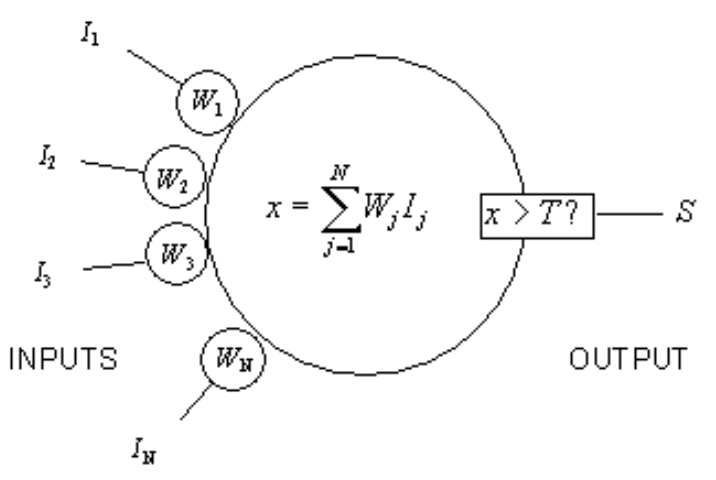

Fig.3: ANN Neuron

Six ANN architectures were investigated by Zabiri et al. (2009) for modeling the process dynamics. It was found that the Nonlinear Autoregressive Recurrent Network with Exogenous inputs and Serial-Parallel (NARXSP) structure gave the 


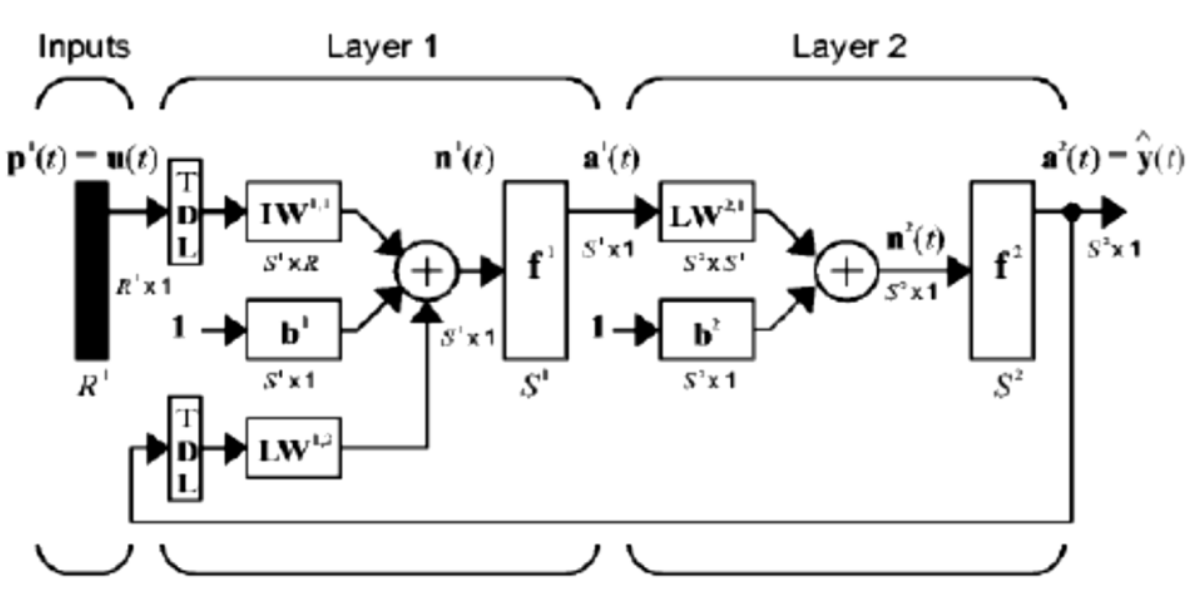

Fig.4: NARX ANN Structure

least Root Mean Square Error (RMSE). Hence, it was chosen as the best ANN architecture to be used for the quantification algorithm. The NARXSP ANN structure is illustrated below in Fig. 4 and Fig.5.

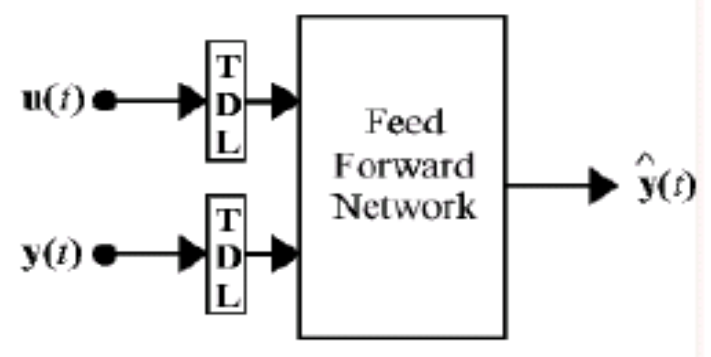

Fig.5: NARX Series - Parallel Structure

\section{Stiction Quantification Algorithm}

The quantification algorithm quantifies the stiction by minimizing the RMSE value. This RMSE minimization is between the actual process variable $P V_{\text {Plant }}$ and the simulated process variable $P V_{\text {model }}$ which is the output from NARXSP ANN. The algorithm is explained in Fig.6 and Fig.7. The quantification algorithm is summarized as follow:

1) The same controller output $O P$ collected from Choudhury's sticky loop is fed to Choudhury's valve stiction model to produce sticky manipulated variable $M V_{n}$ based on initial value of stiction parameters $S$ and $J$.

2) The sticky manipulated variable $M V_{n}$ is fed to NARX to produce process variable $P V_{\text {model }}$ which has stiction effect.

3) The RMSE value is calculated between $P V_{\text {Plant }}$ and $P V_{\text {model }}$.

4) The value of $S$ is changed and new corresponding values of $M V_{n}$ and $P V_{\text {model }}$ are calculated.

5) A new RMSE values between $P V_{\text {Plant }}$ and new $P V_{\text {model }}$ is calculated.

6) The algorithm compares the values of previous RMSE against new RMSE to determine the search direction as follow:

a. If the new RMSE value is greater than old RMSE, then discard $S$ value and choose $S$ values in the opposite direction and calculate all corresponding values of RMSE.

b. If the new RMSE value is smaller than old RMSE, then choose $S$ values in the next $S$ value in the same direction and calculate all 


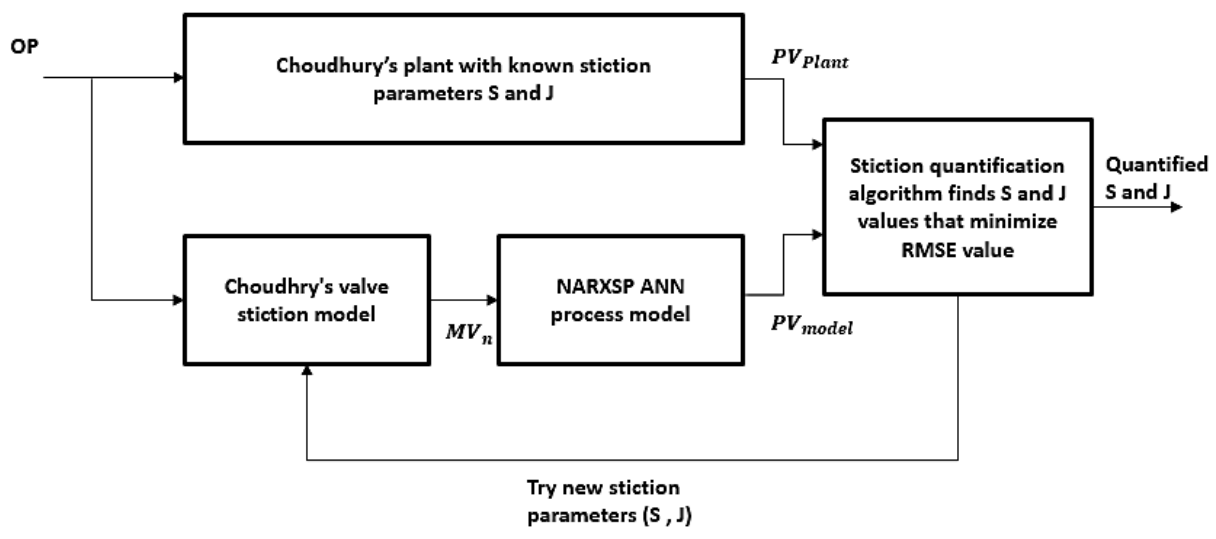

Fig.6: Stiction quantification block diagram

corresponding values of RMSE.

7) Select the $S$ value that produces the least RMSE value.

8) Repeat above steps for J stiction parameter.

9) $S$ and $J$ that produces the least amount of RMSE are the quantified stiction parameters that describe the stiction in the loop.

\section{Research Methodology}

The research methodology flow chart shown in Fig. $\mathbf{8}$ is described as follow:

1) Build Zabiri's algorithm and Choudhury's stiction model and plant in MATLAB and Simulink software.

2) Tune and train NARX Artificial Neural Network to mimic Choudhury's plant dynamics. MATLAB Neural Network Time Series Tool (ntstool) is used for this purpose.

3) Conduct five case studies to test the robustness of Zabiri's stiction quantification algorithm. The five case studies are explained below in section 2.3.

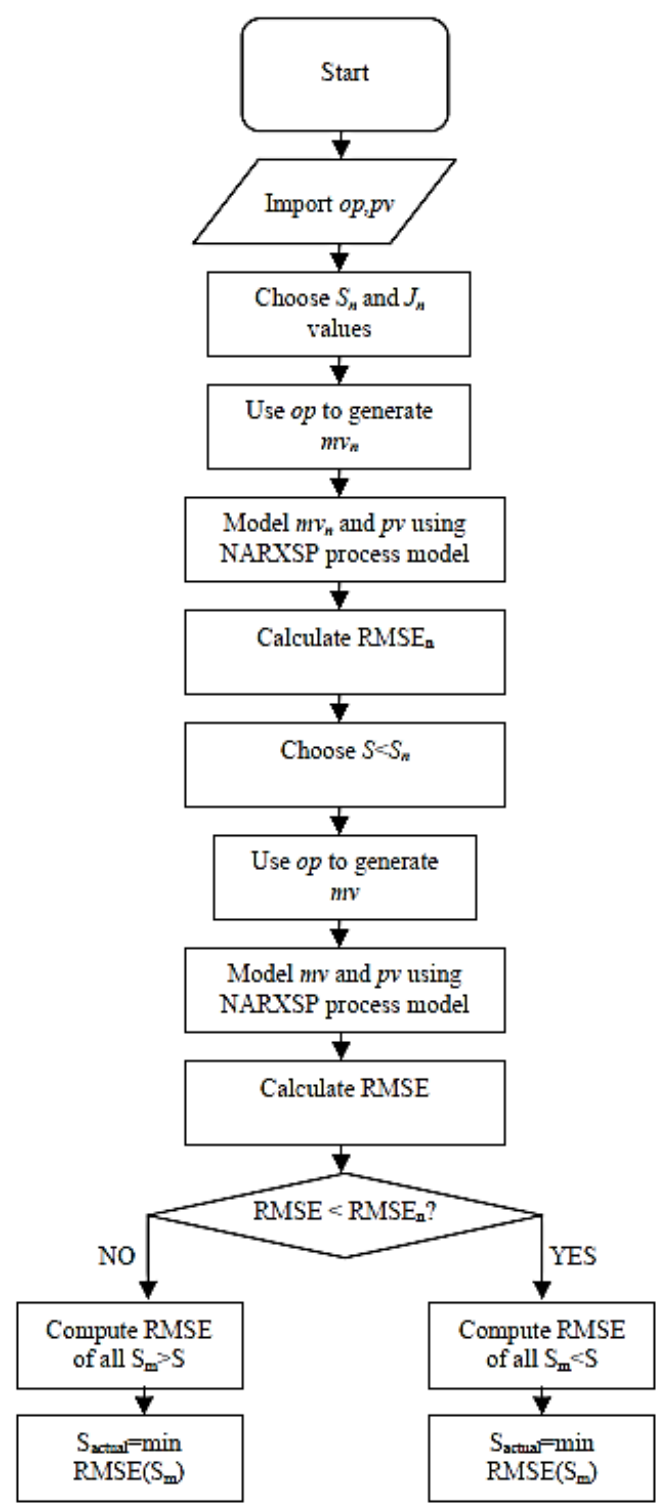

Fig.7: Stiction quantification algorithm adopted from Zabiri et al. (2009). 


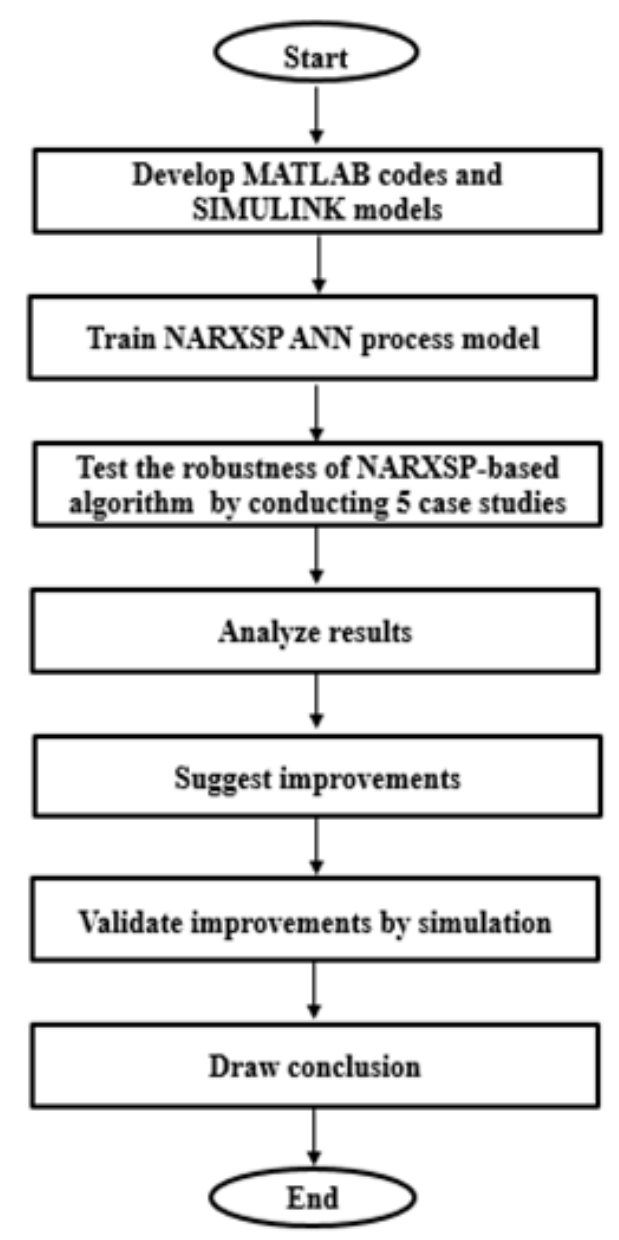

Fig. 8: Research Flow Chart

4) Evaluate and analyze the results from the case studies as explained below in section 2.3.

5) Suggest possible improvements to Zabiri's quantification algorithm.

6) Validate the suggested improvements using simulation.

7) Draw conclusion.

\section{Robustness Tests}

This stiction quantification algorithm in Zabiri et al. (2009) was subjected to stiction value up to $S=3$ and $J=2$ under different controller parameters as mentioned above in Table 2. Therefore, it is worthy to investigate the algorithm performance beyond these operating conditions to check its robustness which is the objective of this research. Hence, the following case studies are conducted:

1- Case Study No.1

It investigates the algorithm performance under stiction overshoot, undershoot and equal stiction in the absence of disturbance. The controller tuning parameters $K_{c}$ and $\tau_{i}$ are set to the most aggressive values i.e. 0.15 and 0.15 respectively.

2- Case Study No.2

It investigates the algorithm performance under stiction overshoot $(S<J)$, undershoot $(S>J)$ and equal stiction $(S=J)$ in the presence of sinusoidal disturbance, noise and time delay. The controller tuning parameters $K_{c}$ and $\tau_{i}$ are set to the most aggressive values i.e. 0.15 and 0.15 respectively.

\section{3- Case Study No.3}

It investigates the impact of incorporating valve resolution on the algorithm performance for stiction overshoot $(S<J)$, undershoot $(S>J)$ and equal stiction $(S=\mathcal{S})$. The controller tuning parameters $K_{c}$ and $\tau_{i}$ are set to the most aggressive values i.e. 0.15 and 0.15 respectively.

\section{4- Case Study No.4}

It investigates the impact of the process model accuracy on the algorithm stiction overshoot $(S<J)$, undershoot $(S$ $>J$ ) and equal stiction $(S=J)$. In this case, the performance of NARX process model is compared against a first order process model. The controller tuning 
parameters $K_{c}$ and $\tau_{i}$ are set to the most aggressive values i.e. 0.15 and 0.15 respectively.

\section{5- Case Study No.5}

It investigates the application of the algorithm on MV feedback travel data rather PV data in case the feedback travel data is available at the plant thorough smart instrumentation.

The above tests results are compared and evaluated based on the following types of errors:

\section{1- Mean Square Error (MSE)}

MSE is used for describing NARX and first order process model accuracies and it is calculated using (3) below.

$$
M S E=\frac{\sum_{1}^{n}\left(y^{\prime}-y\right)^{2}}{n}
$$

\section{2- Root Mean Square Error (RMSE)}

RMSE is used in the quantification algorithm for describing how close is from $P V_{\text {Plant }}$ in each iteration the stiction values $S$ or $J$ is changed. It is calculated using (4) below.

$$
R M S E=\sqrt{\frac{\sum_{1}^{n}\left(y^{\prime}-y\right)^{2}}{n}}
$$

\section{3- Span Error}

It is used to measure the deviation of the quantified stiction values $S$ and $J$ from the actual stiction values (target values) built in Choudhury's plant. It is calculated using (5)

$$
S E=S_{\text {Quantified }}-S_{\text {Actual }}
$$

$$
S E=J_{\text {Quantified }}-J_{\text {Actual }}
$$

\section{4- Absolute Span Error}

It is the absolute value of the span error. It is used to measure the absolute deviation of the quantified stiction values $S$ and $J$ from the actual stiction values (target values) built in Choudhury's plant. It is calculated using (6).

$$
A B S=|S E|
$$

\section{5- Percentage Error}

It is used to measure the deviation between the RMSE values for quantified stiction and target stiction. It is calculated as shown in (7) below.

$$
P E=\frac{\left|R M S E_{1}-R M S E_{2}\right|}{R M S E_{1}} \times 100
$$

\section{RESULTS AND DISCUSSION}

\section{NARX Process Model Training}

The artificial neural network process model (NARX) was trained to imitate the plant dynamics. NARX model has four parameters which are input delay (ID), feedback delay (FD), number of data samples (Ns) and number of hidden neurons (Nn). NARX performance which is measured in mean squared error (MSE) is directly impacted by the tuning of these parameters. It was observed that tuning NARX model was time consuming since it is completely heuristic process. The best parameters combination that produced the lowest MSE i.e. 2.17E-07 was ID $=0$, $\mathrm{FD}=18, \mathrm{Ns}=10,000$ and $\mathrm{Nn}=10$. This 

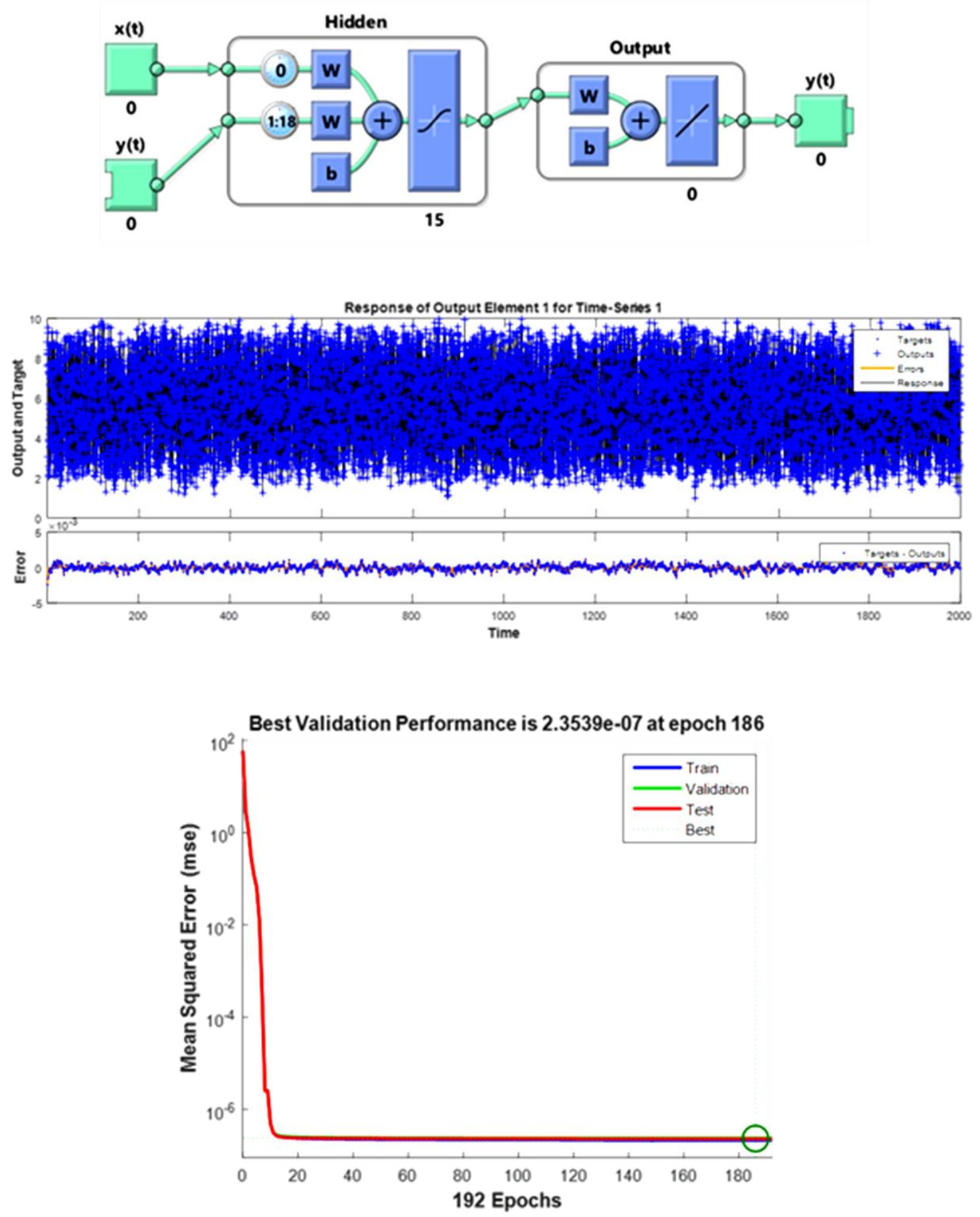

Fig.9: NARX structure and performance

combination was found after more than forty trails. Fig.9 shows NARX structure and its performance.

\section{Case Study No.1}

This case study investigated the algorithm behavior in the absence of disturbance and noise under strong stiction. The algorithm was tested under stiction overshoot $(S<J)$, undershoot $(S>J)$ and equal stiction $(S=J)$. The controller parameters were set to $K_{c}=0.15$ and $\tau_{i}$ $=0.15$. The algorithm managed to quantify the stiction value built in the loop 
Table 3. Stiction quantification results without disturbance

\begin{tabular}{llllllll}
\hline \multirow{2}{*}{ Case Study } & \multicolumn{2}{c}{ Target Value } & \multicolumn{2}{c}{ Quantified Value } & \multicolumn{2}{c}{ Span Error } \\
\cline { 2 - 8 } & S & J & S & J & S & J \\
\hline \multirow{3}{*}{ Undershoot } & 2 & 1 & 2 & 1 & 0 & 0 \\
\cline { 2 - 8 } & 3 & 1 & 4 & 1 & -1 & 0 \\
\hline \multirow{2}{*}{ Overshoot } & 1 & 3 & 1 & 2 & 0 & 1 \\
\hline \multirow{2}{*}{ Equal } & 2 & 4 & 3 & 4 & -1 & 0 \\
\hline & 2 & 2 & 2 & 1 & 0 & 1 \\
\hline
\end{tabular}

Table 4. Stiction undershoot (S2J1)

\begin{tabular}{lllcccl}
\hline No. & $\mathbf{S}$ & $\mathbf{J}$ & $\begin{array}{c}\text { RMSE } \\
(\mathbf{n}=\mathbf{3 0 0})\end{array}$ & $\begin{array}{c}\text { RMSE } \\
(\mathbf{n}=\mathbf{1 5 0 0})\end{array}$ & $\begin{array}{c}\text { RMSE } \\
(\mathbf{n}=\mathbf{1 5 0 0})\end{array}$ & Remark \\
$\mathbf{1}$ & 1 & 1 & 0.8926 & 0.4557 & 0.3859 & \\
\hline $\mathbf{2}$ & 1 & 2 & 0.9800 & 0.5617 & 0.5015 & \\
\hline $\mathbf{3}$ & 1 & 3 & 1.0817 & 0.6858 & 0.6331 & \\
\hline $\mathbf{4}$ & $\mathbf{2}$ & $\mathbf{1}$ & 0.8218 & 0.3766 & $\mathbf{0 . 2 9 7 4}$ & $\begin{array}{l}\text { Quantified and } \\
\text { target value }\end{array}$ \\
\hline $\mathbf{5}$ & 2 & 2 & 0.8865 & 0.4408 & 0.3677 & \\
\hline $\mathbf{6}$ & 2 & 3 & 0.9710 & 0.5419 & 0.4783 & \\
\hline $\mathbf{7}$ & $\mathbf{3}$ & $\mathbf{1}$ & $\mathbf{0 . 7 8 0 7}$ & $\mathbf{0 . 3 7 0 8}$ & 0.3003 & Quantified value \\
\hline $\mathbf{8}$ & 3 & 2 & 0.8188 & 0.3766 & 0.2982 & \\
\hline $\mathbf{9}$ & 3 & 3 & 0.8819 & 0.4395 & 0.3669 & \\
\hline
\end{tabular}

within +/- 1 span error as shown in the Table 3. The detailed results of each case are further discussed in the subsections below.

\section{Stiction Undershoot $(S>J)$}

The quantification algorithm was tested under sticiton undershoot scenario where the stiction parameters set to stickband $S=2$ and slip-jump $J=1$. The quantification result based on RMSE value is shown in Table 4.

The algorithm estimated the amount of stiction as S3J1 rather than S2J1 which is the actual value built in the simulation model. It was found that the algorithm produced smaller value of RMSE i.e. 0.3708 for S3J1 stiction whereas RMSE of 0.3766 for S2J1 that is the actual value of stiction as shown above in Table 4 . It is worth noting that the percentage error between the two RMSE values is $1.5 \%$.

It was found that the RMSE calculation which is the core of the quantification algorithm improves by increasing the number of sampling points used in simulation. Hence, by increasing the number of the sampling points to 2500 points the calculation of the RMSE improved and the algorithm succeeded in quantifying the right amount of stiction which is S2J1. 
Table 5. Stiction Overshoot (S1J3)

\begin{tabular}{cccccl}
\hline No. & $\mathbf{S}$ & $\mathbf{J}$ & $\begin{array}{c}\text { RMSE } \\
(\mathbf{n}=\mathbf{2 5 0 0})\end{array}$ & $\begin{array}{c}\text { RMSE } \\
(\mathbf{n = 5 0 0 0 )}\end{array}$ & Remark \\
\hline $\mathbf{1}$ & 1 & 1 & 0.3799 & 0.3371 & \\
$\mathbf{2}$ & $\mathbf{1}$ & $\mathbf{2}$ & $\mathbf{0 . 3 3 4 6}$ & $\mathbf{0 . 2 7 9 8}$ & Quantified value \\
\hline $\mathbf{3}$ & 1 & 3 & 0.3451 & 0.2870 & Target value \\
\hline $\mathbf{4}$ & 1 & 4 & 0.4072 & 0.3548 & \\
\hline $\mathbf{5}$ & 2 & 1 & 0.4982 & 0.4711 & \\
\hline $\mathbf{6}$ & 2 & 2 & 0.4543 & 0.4232 & \\
\hline $\mathbf{7}$ & 2 & 3 & 0.4519 & 0.4191 & \\
\hline $\mathbf{8}$ & 2 & 4 & 0.4915 & 0.4601 & \\
\hline $\mathbf{9}$ & 3 & 1 & 0.6468 & 0.6284 & \\
\hline $\mathbf{1 0}$ & 3 & 2 & 0.7306 & 0.7161 & \\
\hline $\mathbf{1 1}$ & 3 & 3 & 0.8444 & 0.8337 & \\
\hline $\mathbf{1 2}$ & 3 & 4 & 0.9778 & 0.9703 & \\
\hline $\mathbf{1 3}$ & 4 & 1 & 0.6058 & 0.5843 & \\
\hline $\mathbf{1 4}$ & 4 & 2 & 0.6474 & 0.6288 & \\
\hline $\mathbf{1 5}$ & 4 & 3 & 0.7315 & 0.7166 & \\
\hline $\mathbf{1 6}$ & 4 & 4 & 0.8455 & 0.8342 & \\
\hline
\end{tabular}

\section{Stiction Overshoot $(S<J)$}

The quantification algorithm was tested under stiction overshoot scenario where the stiction parameters set to stickband $S=1$ and slip-jump $J=3$. The quantification result is shown in Table $\mathbf{5}$.

The stickband parameter (S) value was quantified correctly as 1 whereas the slipjump parameter (J) was quantified as 2 instead of 3 . The number of samples was increased from 2,500 points to 5,000 points in order to improve the RMSE value for improving the quantification result. The algorithm percentage error between the RMSE value for 2,500 points and 5,000 points dropped from $3.1 \%$ to $2.6 \%$, however, the stiction quantification result did not change. The span error in the slipjump $(J)$ which is -1 is attributed to NARX model error as investigated in Case Study No.4.

\section{Equal Stiction $(S=J)$}

The quantification algorithm is tested under equal stiction scenario where the stiction parameters set to stickband $\mathrm{S}=4$ and slip-jump $J=4$. The quantification result is shown in Table 6 .

The stickband value (S) was quantified as 5 instead of 4 with 1 unit difference whereas the slip-jump (J) was quantified correctly as 4. The RMSE value for quantified stiction S5J4 was found to be 0.3054 against 0.4019 RMSE value for the actual amount of stiction i.e. S4J4. The span error in stickband (S) i.e. +1 is attributed to the NARX model error as investigated in Case Study No.4 below.

\section{Case Study No.2}

A low frequency sinusoidal disturbance and noise sources were added to the loop as shown in Fig.10. The amplitude and the 
Table 6. Equal Stiction (S4J4)

\begin{tabular}{|c|c|c|c|c|}
\hline No. & $\mathbf{S}$ & J & $\begin{array}{c}\text { RMSE } \\
(n=2500)\end{array}$ & Remark \\
\hline 1 & 1 & 1 & 0.5596 & \\
\hline 2 & 1 & 2 & 0.6628 & \\
\hline 3 & 1 & 3 & 0.7812 & \\
\hline 4 & 1 & 4 & 0.9089 & \\
\hline 5 & 1 & 5 & 1.0424 & \\
\hline 6 & 2 & 1 & 0.4705 & \\
\hline 7 & 2 & 2 & 0.5314 & \\
\hline 8 & 2 & 3 & 0.6231 & \\
\hline 9 & 2 & 4 & 0.7342 & \\
\hline 10 & 2 & 5 & 0.8570 & \\
\hline 11 & 3 & 1 & 0.4409 & \\
\hline 12 & 3 & 2 & 0.4348 & \\
\hline 13 & 3 & 3 & 0.4782 & \\
\hline 14 & 3 & 4 & 0.5596 & \\
\hline 15 & 3 & 5 & 0.6652 & \\
\hline 16 & 4 & 1 & 0.4845 & \\
\hline 17 & 4 & 2 & 0.4067 & \\
\hline 18 & 4 & 3 & 0.3756 & \\
\hline 19 & 4 & 4 & 0.4019 & Target value \\
\hline 20 & 4 & 5 & 0.4763 & \\
\hline 21 & 5 & 1 & 0.5860 & \\
\hline 22 & 5 & 2 & 0.4638 & \\
\hline 23 & 5 & 3 & 0.3630 & \\
\hline 24 & 5 & 4 & 0.3054 & Quantified Value \\
\hline 25 & 5 & 5 & 0.3156 & \\
\hline
\end{tabular}

frequency of the sine wave were set to 0.5 and $6 \mathrm{rad} / \mathrm{s}$ respectively. The remaining test conditions were maintained as same as in Case Study No.1. The quantification algorithm was tested under stiction overshoot $(\mathrm{S}<\mathrm{J})$, stiction undershoot $(\mathrm{S}>\mathrm{J})$ and equal stiction $(S=J)$. The controller parameters were set to $K_{c}=0.15$ and $\tau_{i}$ $=0.15$. It was found that the algorithm managed to quantify the stiction value built in the loop within +/- 1 span error as shown in the Table 7. NARX output versus plant output under disturbance is shown in Fig.11.
The disturbance frequency and amplitude were increased by 4 folds in order to further check the algorithm robustness against disturbance. It was observed that the algorithm produced the same quantification result as in Table 7 despite the increment in disturbance. It is concluded that the quantification algorithm is robust against disturbance and noise since it manged to produce a similar result to Case Study No. 1 which is without disturbance.

Furthermore, the impact of process time delay in the control loop on the 

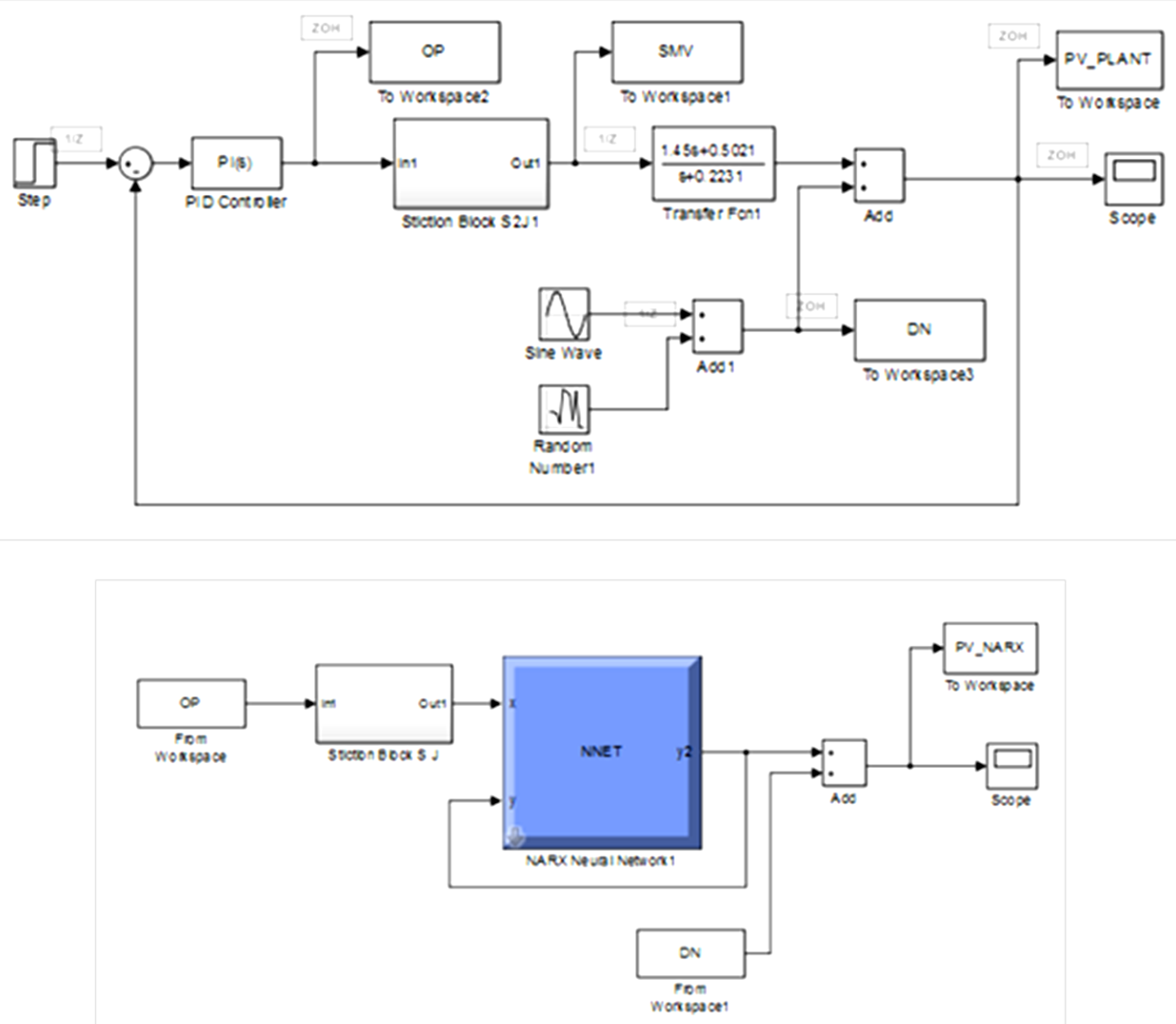

Fig.10: Simulink plants with disturbance and noise

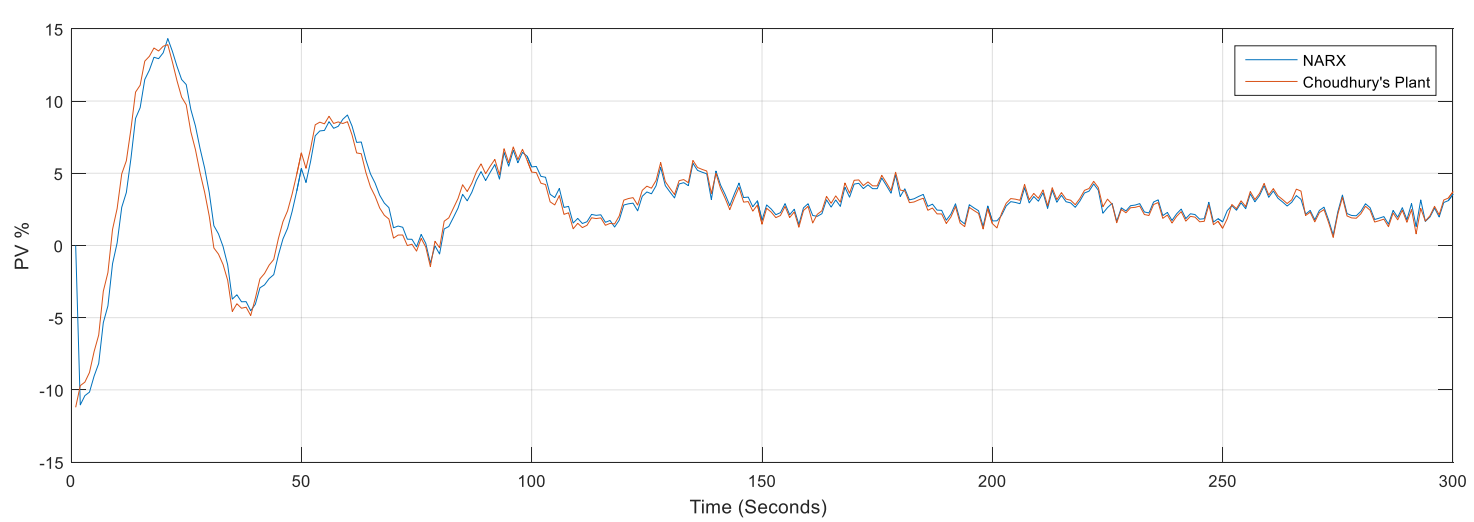

Fig.11: Plant and NARX PV under noise and disturbance

quantification algorithm was investigated. It was observed that the control loop becomes marginally stable with sustained oscillation by adding 3 seconds time delay as shown below in Fig.12 under current controller settings in addition to stiction S2J1. The Simulink models are shown in Fig.13.

The algorithm quantified the stiction values with span error of $+/-1$ in 
Table 7. Stiction quantification results under disturbance and noise

\begin{tabular}{lcccccc}
\hline \multirow{2}{*}{ Case Study } & \multicolumn{2}{c}{ Target Value } & \multicolumn{2}{c}{ Quantified Value } & \multicolumn{2}{c}{ Span Error } \\
\cline { 2 - 7 } & $\mathbf{S}$ & J & S & J & S & J \\
\hline \multirow{2}{*}{ Undershoot } & 2 & 1 & 2 & 1 & 0 & 0 \\
\hline \multirow{2}{*}{ Overshoot } & 3 & 1 & 4 & 1 & -1 & 0 \\
\hline \multirow{2}{*}{ Equal } & 1 & 3 & 1 & 2 & 0 & 1 \\
\hline & 2 & 4 & 2 & 3 & 0 & -1 \\
\hline & 2 & 2 & 2 & 1 & 0 & 1 \\
\hline
\end{tabular}

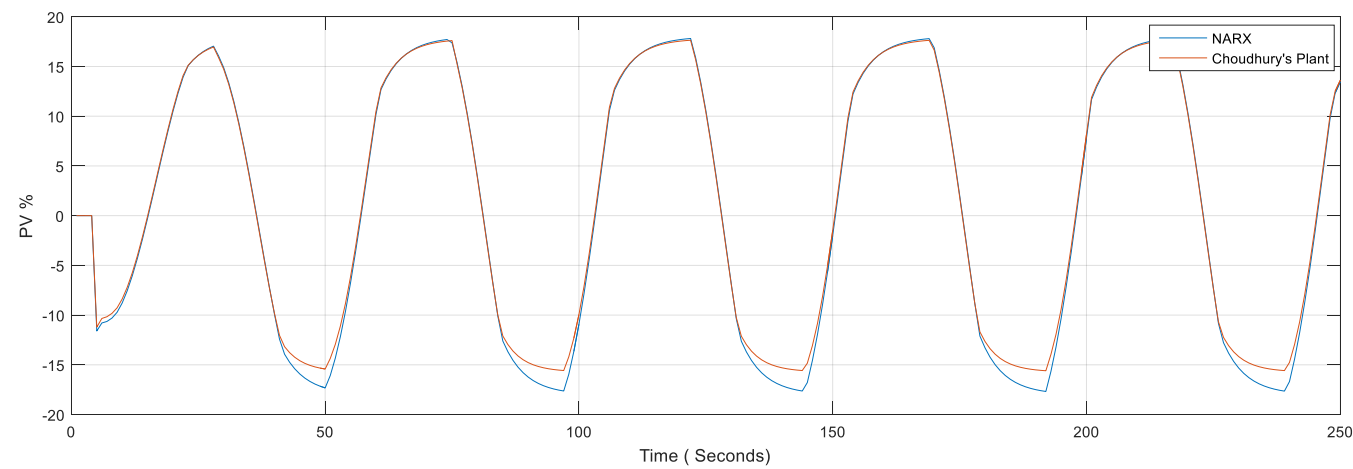

Fig.12: Time delay impact
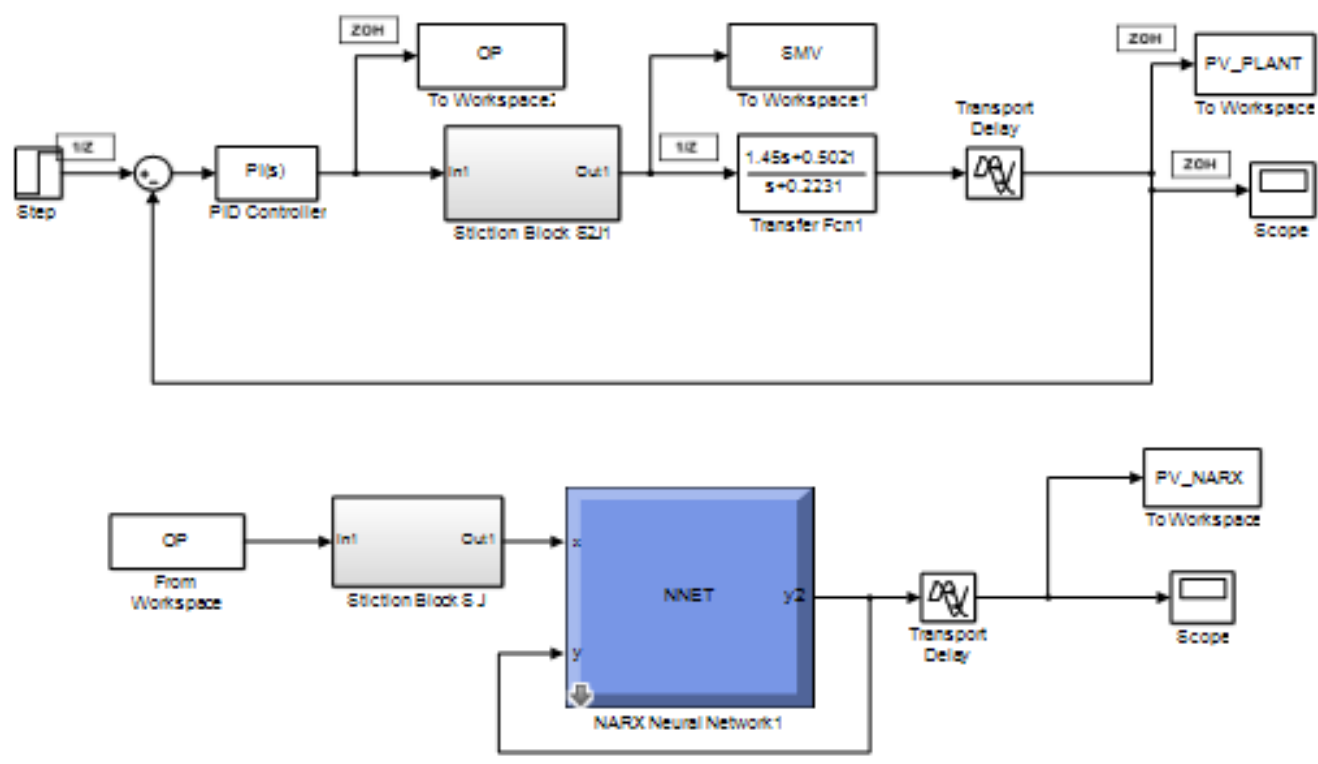

Fig.13: Plants with time delay

overshoot and equal stiction cases, which is similar to the quantification results when the loop had no time delay. Hence, the algorithm is not affected by the presence of time delay in the loop since it managed to produce the same results in marginally stable condition. However, the span error increased from $+/-1$ to $+/-2$ in the case of 
Table 8. Quantification results under process time delay

\begin{tabular}{lcccccc}
\hline \multirow{2}{*}{ Case Study } & \multicolumn{2}{c}{ Target Value } & \multicolumn{2}{c}{ Quantified Value } & \multicolumn{2}{c}{ Span Error } \\
\cline { 2 - 7 } & S & J & S & J & S & J \\
\hline \multirow{2}{*}{ Undershoot } & 2 & 1 & 3 & 1 & -1 & 0 \\
\cline { 2 - 7 } Overshoot & 3 & 1 & 4 & 1 & -1 & 0 \\
\hline \multirow{2}{*}{ Equal } & 1 & 3 & 3 & 4 & -2 & -1 \\
\hline & 2 & 4 & 3 & 4 & -1 & 0 \\
\hline
\end{tabular}

Table 9. Stiction undershoot under quantification with resolution

\begin{tabular}{ccccccc}
\hline \multirow{2}{*}{ Resolution } & \multicolumn{2}{c}{ Target Value } & \multicolumn{2}{c}{ Quantified Value } & \multicolumn{2}{c}{ Span Error } \\
\cline { 2 - 7 } & S & J & S & J & S & J \\
\hline 1 & 2.7 & 1.5 & 3 & 1 & -0.3 & 0.5 \\
\hline 0.8 & 2.7 & 1.5 & 3.2 & 1.6 & -0.5 & -0.1 \\
\hline 0.75 & 2.7 & 1.5 & 3.75 & 2.25 & -1.05 & -0.75 \\
\hline 0.6 & 2.7 & 1.5 & 3.6 & 1.8 & -0.9 & -0.3 \\
\hline 0.5 & 2.7 & 1.5 & 3.5 & 2 & -0.8 & -0.5 \\
\hline 0.2 & 2.7 & 1.5 & 3.6 & 2 & -0.9 & -0.5 \\
\hline 0.1 & 2.7 & 1.5 & 3.3 & 1.6 & -0.6 & -0.1 \\
\hline
\end{tabular}

stiction overshoot specifically when $S=1$ and $J=3$ as shown in Table 8 . This span error increment in case of stiction overshoot is attributed to the error in NARX model. The impact of model accuracy used in quantification algorithm is investigated in Case Study No.5 below.

\section{Case Study No.3}

In industry, the values of stiction parameters $S$ and $J$ in actual control loop can be any real number and not necessarily integers. Therefore, the algorithm and subsequently MATLAB code were modified to incorporate valve and search resolutions. Hence, different values of valve resolution were tested in an attempt to reduce the quantification error.
In this case study, the algorithm was tested under stiction undershoot $(\mathrm{S}>\mathrm{J})$ and equal stiction $(S=J)$ scenarios. It was expected that as the search resolution value gets smaller the span error reduces as well. However, it was observed that there was no clear reduction trend in the span error. For instance, in stiction undershoot case, the absolute span error of $J$ parameter is 0.1 for resolution value equal to 0.8 whereas the absolute span error is 0.5 for resolution value equal to 0.2 as shown in Table 9.

In equal stiction case, the same behavior was observed whereby the absolute span error for J parameter is 0 for resolution value equal to 1 , whereas the absolute span error is 0.9 when the 
Table 10. Equal stiction quantification with resolution

\begin{tabular}{ccccccc}
\hline \multirow{2}{*}{ Resolution } & \multicolumn{2}{c}{ Target Value } & \multicolumn{2}{c}{ Quantified Value } & \multicolumn{2}{c}{ Span Error } \\
\cline { 2 - 7 } & S & J & S & J & S & J \\
\hline 1 & 4 & 4 & 5 & 4 & -1 & 0 \\
\hline 0.8 & 4 & 4 & 4.8 & 4 & -0.8 & 0 \\
\hline 0.75 & 4 & 4 & 4.5 & 3.75 & -0.5 & 0.25 \\
\hline 0.6 & 4 & 4 & 4.8 & 4.2 & -0.8 & -0.2 \\
\hline 0.5 & 4 & 4 & 5 & 4.5 & -1 & -0.5 \\
\hline 0.2 & 4 & 4 & 4.8 & 4.2 & -0.8 & -0.2 \\
\hline 0.1 & 4 & 4 & 4.3 & 4.9 & -0.3 & -0.9 \\
\hline 0.01 & 4 & 4 & 4.9 & 4.3 & -0.9 & -0.3 \\
\hline
\end{tabular}

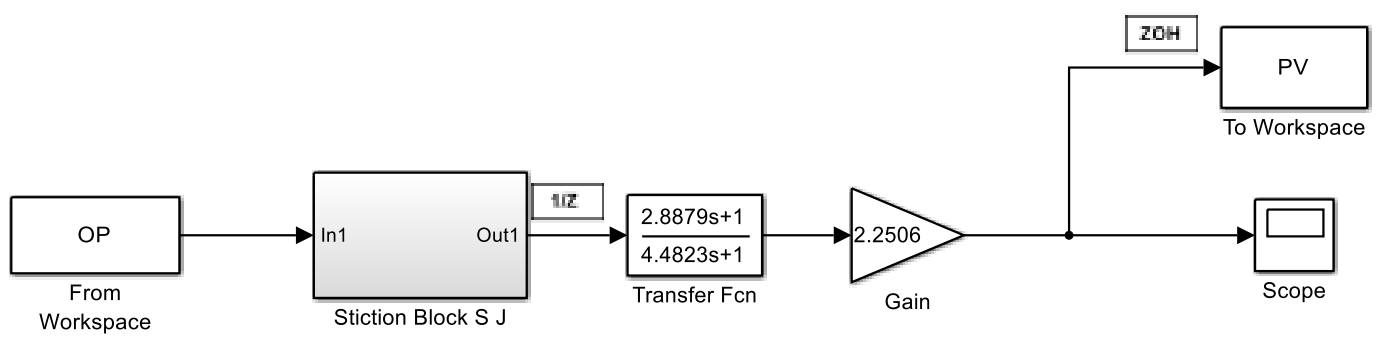

Fig.14: First Order Model Plant

resolution decreased to 0.1 . The error results versus the resolution values are shown in Table 10. The unsystematic error trend is attributed to the error between NARX model and plant model. Therefore, the impact of model accuracy on quantification error is investigated in Case Study No. 5 below.

\section{Case Study No.4}

This case study investigates the impact of model accuracy on quantification error. The plant dynamics was modeled as first order process model as shown in (8) and Fig.14.

$$
G_{P}(s)=\frac{2.2506(1+2.8879 S)}{(1+4.4823 S)}
$$

The first order mean squared error is $3.158 \mathrm{e}-18$ which is very accurate in describing the plant dynamics when compared with NARX model error i.e. 2.174e-07. It was observed that the quantification algorithm managed to quantify precisely the stiction value with RMSE approximately equal to 0 and 0 span error. Unlike NARX model which has relatively large RMSE and +/- 1 span error. The quantification results with the span error for different values of stiction are 
Table 11. Quantification results from high accuracy model

\begin{tabular}{lllcl}
\hline No. & $\mathbf{S}$ & J & $\begin{array}{c}\text { RMSE } \\
(\mathbf{n = 2 5 0 0 )}\end{array}$ & Remarks \\
\hline $\mathbf{1}$ & 1 & 1 & 0.28138 & \\
\hline $\mathbf{2}$ & 1 & 2 & 0.14068 & Target and quantified values \\
$\mathbf{3}$ & $\mathbf{1}$ & $\mathbf{3}$ & $\mathbf{0 . 0 0 0 0 3}$ & \\
\hline $\mathbf{4}$ & 1 & 4 & 0.14071 & \\
\hline $\mathbf{5}$ & 2 & 1 & 0.47424 & \\
\hline $\mathbf{6}$ & 2 & 2 & 0.44545 & \\
\hline $\mathbf{7}$ & 2 & 3 & 0.45995 & \\
\hline $\mathbf{8}$ & 2 & 4 & 0.51410 & \\
\hline $\mathbf{9}$ & 3 & 1 & 0.61038 & \\
\hline $\mathbf{1 0}$ & 3 & 2 & 0.69501 & \\
\hline $\mathbf{1 1}$ & 3 & 3 & 0.81086 & \\
\hline $\mathbf{1 2}$ & 3 & 4 & 0.94653 & \\
\hline $\mathbf{1 3}$ & 4 & 1 & 0.57158 & \\
\hline $\mathbf{1 4}$ & 4 & 2 & 0.61174 & \\
\hline $\mathbf{1 5}$ & 4 & 3 & 0.69694 & \\
\hline $\mathbf{1 6}$ & 4 & 4 & 0.81314 & \\
\hline
\end{tabular}

Table 12. Quantification results summary

\begin{tabular}{ccccccc}
\hline \multirow{2}{*}{ Case Study } & \multicolumn{2}{c}{ Target Value } & \multicolumn{2}{c}{ Quantified Value } & \multicolumn{2}{c}{ Span Error } \\
\cline { 2 - 8 } & S & J & S & J & S & J \\
\hline Undershoot & 3 & 1 & 3 & 1 & 0 & 0 \\
\hline Overshoot & 1 & 3 & 1 & 3 & 0 & 0 \\
\hline Equal & 4 & 4 & 4 & 4 & 0 & 0 \\
\hline
\end{tabular}

Table 13. Quantification results from high accuracy model with resolution

\begin{tabular}{ccccccc}
\hline \multirow{2}{*}{ Resolution } & \multicolumn{2}{c}{ Target Value } & \multicolumn{2}{c}{ Quantified Value } & \multicolumn{2}{c}{ Span Error } \\
\cline { 2 - 8 } & $\mathbf{S}$ & $\mathbf{J}$ & $\mathbf{S}$ & $\mathbf{J}$ & $\mathbf{S}$ & J \\
\hline $\mathbf{1}$ & 1.6 & 2.2 & 2 & 2 & -0.4 & 0.2 \\
\hline $\mathbf{0 . 5}$ & 1.6 & 2.2 & 1.5 & 2 & 0.1 & 0.2 \\
\hline $\mathbf{0 . 2}$ & 1.6 & 2.2 & 1.6 & 2.2 & 0 & 0 \\
\hline
\end{tabular}

shown in Table 11 and Table 12.

In addition, the impact of using different search resolution values on the quantification accuracy was investigated. It was observed there was a clear reduction trend in error when the search resolution was reduced. For instance, stickband target value was set to $S=1.6$, the quantified value improved from 2 then to 1.5 then to 1.6 as the search resolution was reduced from 1 to 0.5 to 0.2 respectively. The quantification results for different resolution values are shown in Table 13. In turn, it is concluded that using a model with higher accuracy eliminates the quantification span errors 


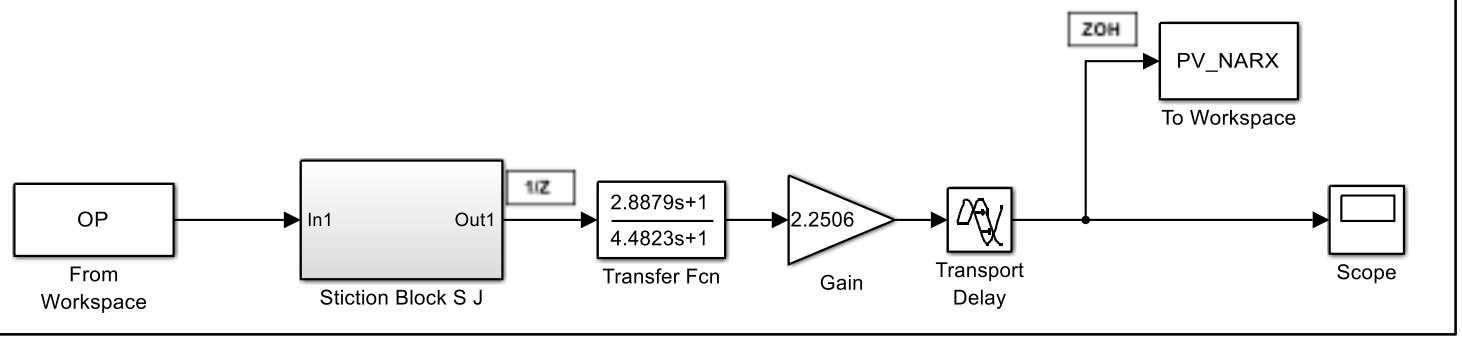

Fig.15: FOPTD Plant

encountered in the previous case studies. In addition, it is concluded that decreasing the search resolution reduces the quantification error in case the stiction parameters $\mathrm{S}$ and $\mathrm{J}$ are real values.

In addition, the impact of time delay on the quantification algorithm was investigated. A time delay of 3 seconds was added to the first order plant as shown in Fig.15. It was observed that the quantification algorithm was not affected by the addition of the time delay. The algorithm quantified the stiction values exactly as in the case study without time delay as shown above in Table 12. As conclusion, the quantification algorithm was not affected by the time delay despite the fact that the time delay made the control loop marginally stable.

\section{Case Study No.5}

It was observed from the previous case studies that the quantification algorithm depends on the availability of an accurate process model. The process model has to be capable of describing the plant dynamics without stiction with relatively very small error to get good stiction estimates. Therefore the need arises for a stiction quantification algorithm that does not depend on the process model in finding the correct stiction values in the loop.

A different quantitation approach that uses the actual valve travel feedback was investigated. This approach does not require the availability of the process model. Rather, it requires the availability of the valve travel feedback at the process control system. As mentioned above in the literature, nowadays smart instrumentation such as Digital Valve Controllers (DVC) or valve smart positioners can provide valve travel feedback through HART or Foundation Fieldbus communication protocol without the need for additional hardware.

The proposed quantification approach relies on the same quantification algorithm to find stiction values. However, the algorithm utilizes the sticky actual feedback travel as shown in Fig.16 to calculate the RMSE value for finding the right stiction parameters $S$ and $J$ that minimizes RMSE. The Simulink model is used for this approach is shown in Fig.17.

This approach was tested under several scenarios as in previous case studies. It was tested under stiction overshoot, undershoot and equal section. In addition, 


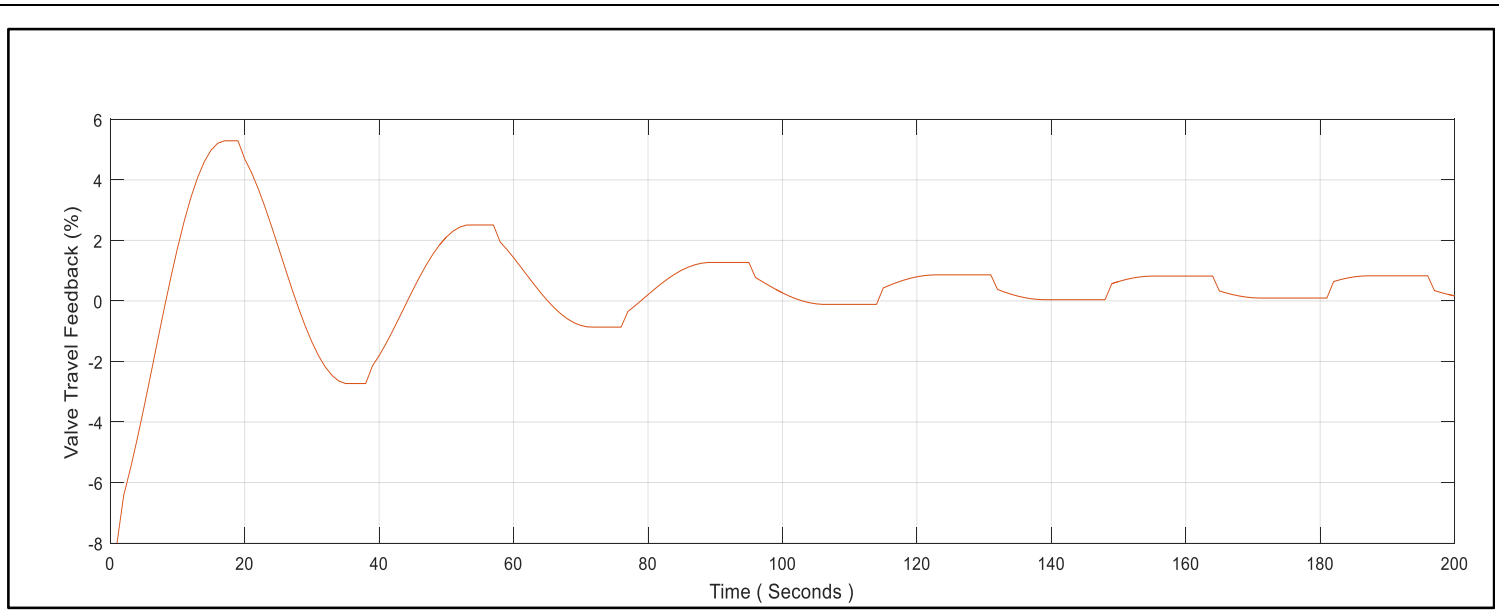

Fig.16: Valve travel feedback with stiction S2J3
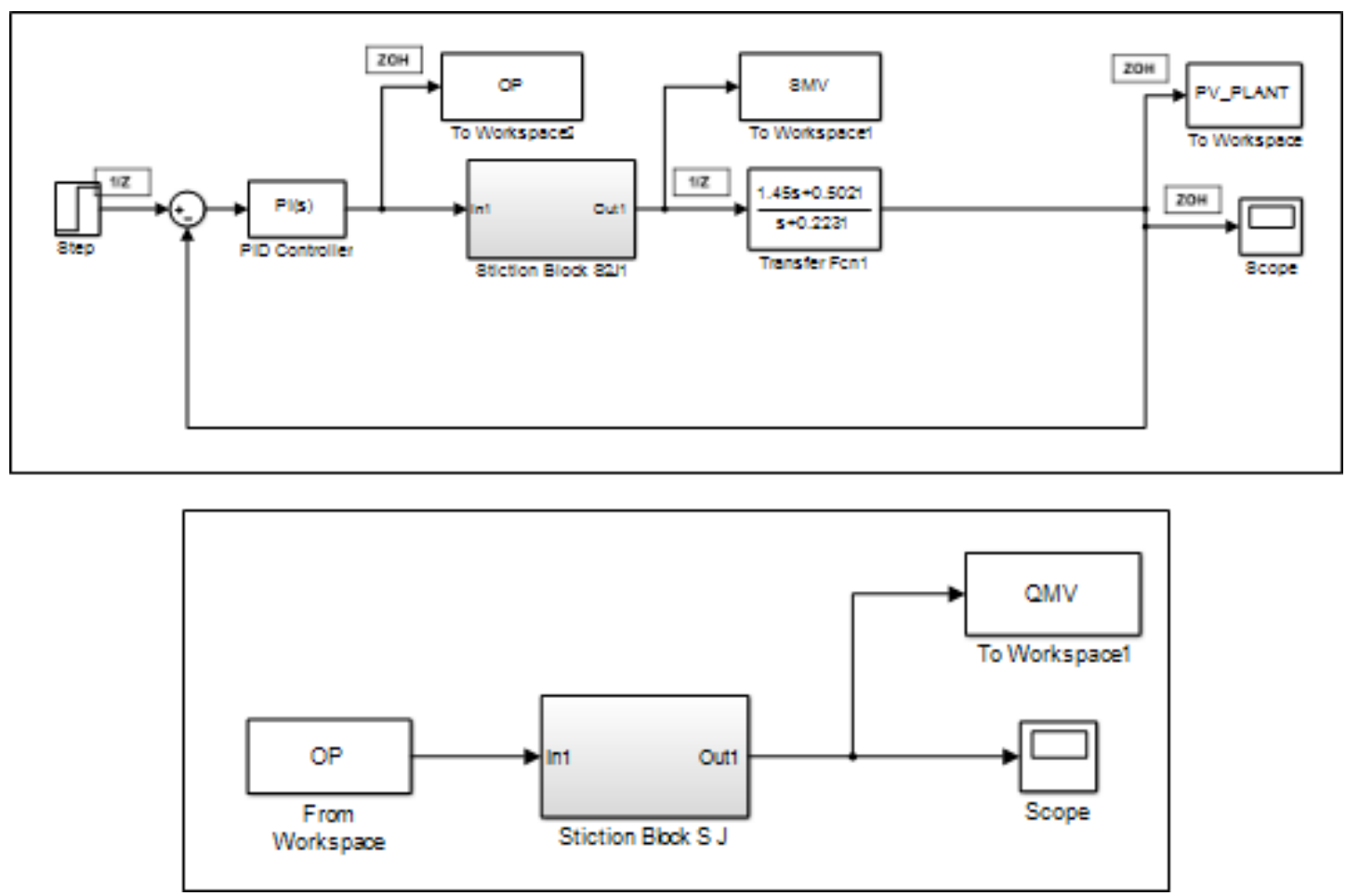

Fig.17: Quantification based on MV data

the impact of the search resolution was also checked. It was observed that the algorithm successfully quantified the stiction with zero span error. Moreover, there was a clear reducing trend in error as the resolution value was reduced. As conclusion, this approach achieved the same accurate results as in case study no.
4 without the need for process model at all. The results are shown in Table 14 and 15.

\section{Improvements}

The summary of improvements on the existing quantification algorithm is summarized as follow: 
Table 14. Quantification results based on MV data

\begin{tabular}{ccccccc}
\hline \multirow{2}{*}{ Case Study } & \multicolumn{2}{c}{ Target Value } & \multicolumn{2}{c}{ Quantified Value } & \multicolumn{2}{c}{ Span Error } \\
\cline { 2 - 8 } & S & J & S & J & S & J \\
\hline \multirow{2}{*}{ Undershoot } & 2 & 1 & 2 & 1 & 0 & 0 \\
\cline { 2 - 8 } & 3 & 1 & 3 & 1 & 0 & 0 \\
\hline \multirow{2}{*}{ Overshoot } & 1 & 3 & 1 & 3 & 0 & 0 \\
\cline { 2 - 8 } & 2 & 4 & 2 & 4 & 0 & 0 \\
\hline \multirow{2}{*}{ Equal } & 2 & 2 & 2 & 2 & 0 & 0 \\
\cline { 2 - 8 } & 4 & 4 & 4 & 4 & 0 & 0 \\
\hline
\end{tabular}

Table 15. Quantification results with different resolutions

\begin{tabular}{ccccccc}
\hline \multirow{2}{*}{ Resolution } & \multicolumn{2}{c}{ Target Value } & \multicolumn{2}{c}{ Quantified Value } & \multicolumn{2}{c}{ Span Error } \\
\cline { 2 - 7 } & $\mathbf{S}$ & $\mathbf{J}$ & $\mathbf{S}$ & $\mathbf{J}$ & $\mathbf{S}$ & J \\
\hline $\mathbf{1}$ & 1.6 & 2.2 & 2 & 2 & -0.4 & 0.2 \\
\hline $\mathbf{0 . 5}$ & 1.6 & 2.2 & 1.5 & 2 & 0.1 & 0.2 \\
\hline $\mathbf{0 . 2}$ & 1.6 & 2.2 & 1.6 & 2.2 & 0 & 0 \\
\hline
\end{tabular}

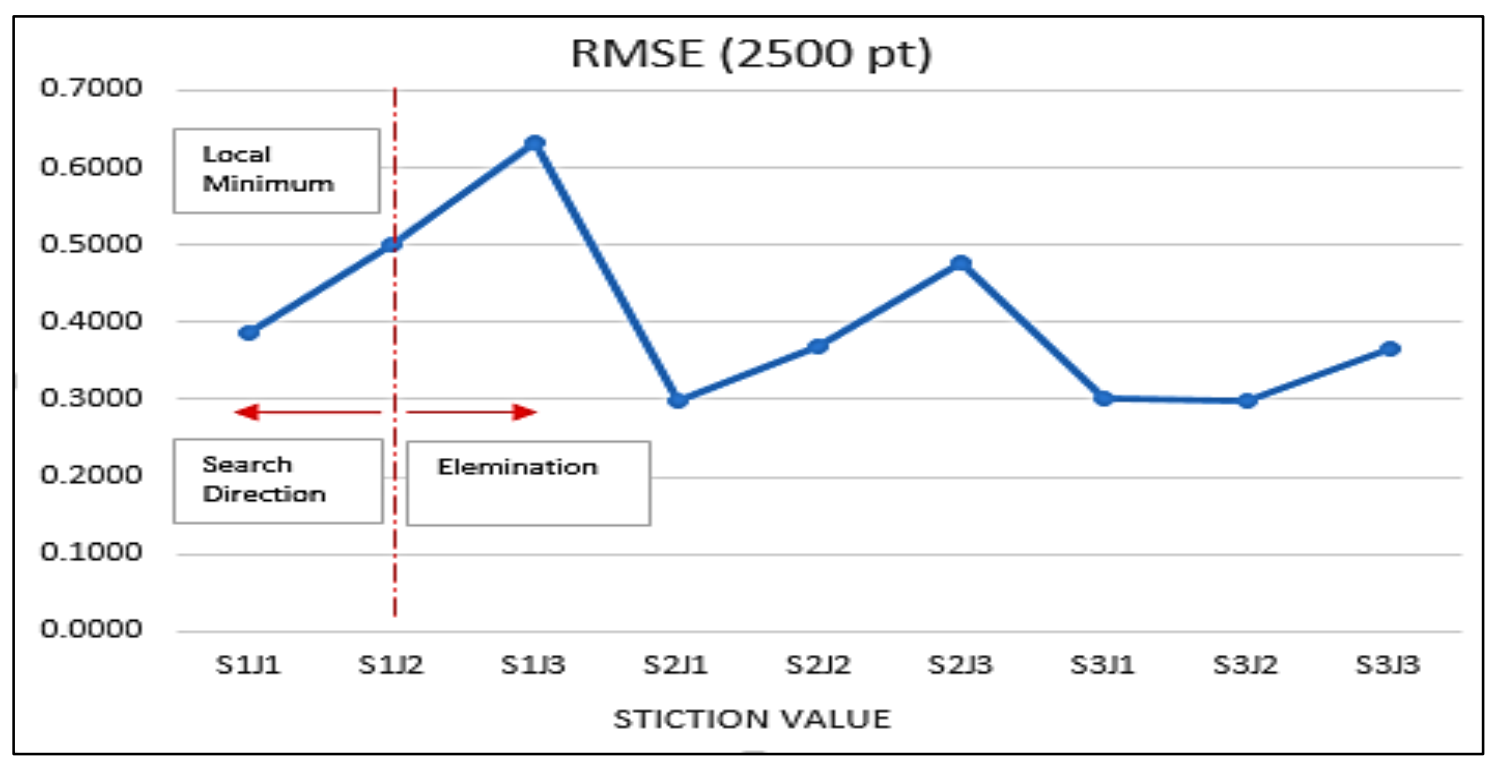

Fig.18: Local Minimum

\section{Elimination of Local Minimum}

It was observed that the current algorithm has a search direction based on the RMSE value as explained in methodology in chapter 3. Such approach might make the algorithm quantify the stiction parameters based on RMSE local minimum which leads to wrong quantification as shown in Fig.18. Hence, this issue can be resolved by calculating the RMSE values for all possible combinations of stiction parameters $S$ and 
J regardless of error direction. The modified algorithm is shown in Fig.19.

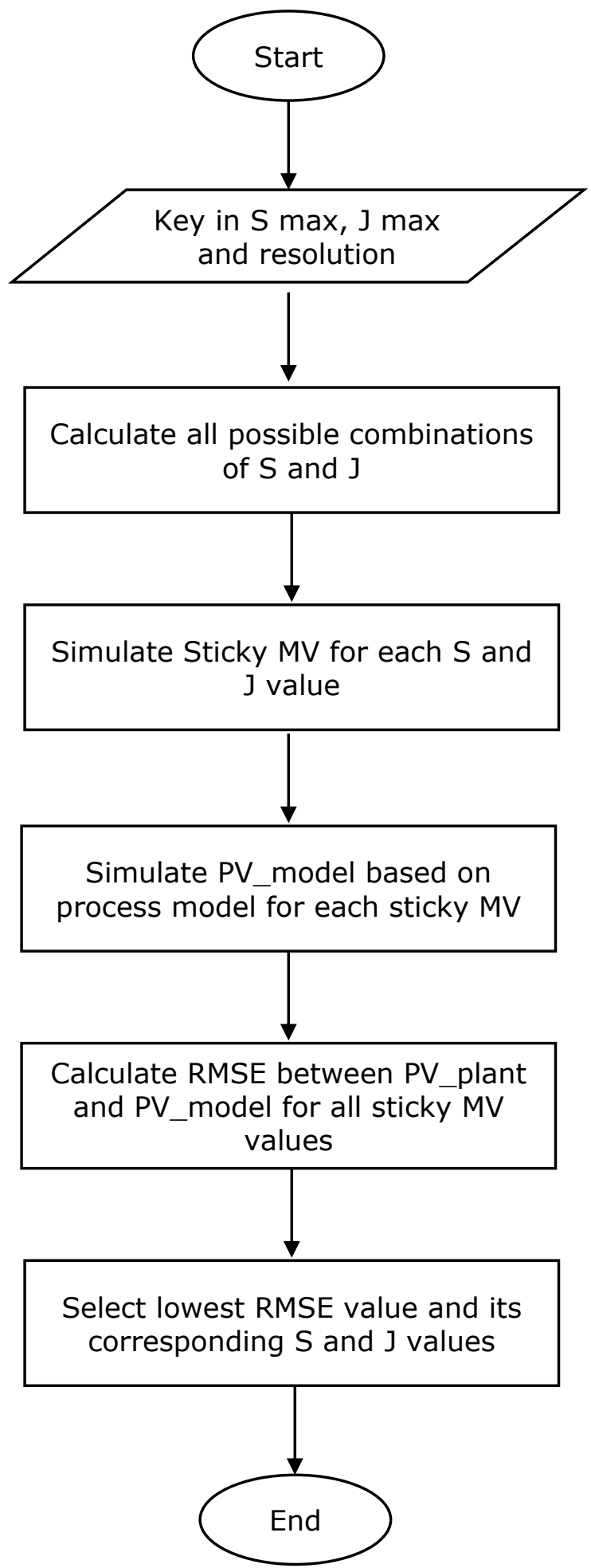

Fig.19: Modified Algorithm Flow Chart

\section{Incorporation of Valve and Search Solutions}

As shown in Case Study No.3, including valve and search resolution improved the algorithm accuracy since the stiction in plant can be any real number rather than integers. It was shown that the span error was reduced by reducing the resolution.

\section{Algorithm Simplification by Using FOPTD}

It was shown in Case Study No.4 that using FOPTD eliminated the span error encountered while using NARX process model since it has higher MSE value. In addition, getting an accurate FOPTD model is much faster than getting an accurate NARX model since tuning NARX model is heuristic.

\section{Elimination of Process Model Dependency}

It was shown in Case Sturdy No.5 that the same algorithm can be applied on MV data rather than PV data in order to eliminate process model dependency.

\section{CONCLUSION RECOMMENDATION}

AND

Five case studies were conducted the stiction quantification algorithm developed by Zabiri et al. The objectives are to test the robustness of the algorithm in several operating conditions and provide improvements. These operating conditions covered aggressive controller tuning, stiction values ranging from weak 
to strong stiction in the presence of disturbance, noise and time delay. In addition, the impact of process model accuracy and algorithm search resolution were investigated. Finally, the algorithm performance was tested on OP-MV data.

It was observed that tuning and training NARX process model is time consuming process since it is heuristic in nature. The best parameters combination that produced the lowest MSE i.e. 2.17E-07 is $I D=0, F D=18, \mathrm{Ns}=10,000$ and $\mathrm{Nn}=$ 10 , which took more than forty trails to be found. The algorithm with NARX process model quantified the stiction parameters the stickband $(\mathrm{S})$ and slip-jump (J) under above operating conditions with absolute span error equal to 1 . This value of span error was sustained under all operating conditions. It is worth noting that the number of data samples used is 2500 points which has direct impact on the RMSE calculation that is the core of the quantification algorithm.

The impact of process model accuracy on the quantification algorithm was investigated. A first order process model with MSE equal to $3.158 \mathrm{e}-18$ which is lower than NARX model was used to describe the plant dynamics. It was observed that the span error was completely eliminated and the stiction parameters were quantified correctly. It was noticed that different combination of stiction parameters result in very similar PV behaviors that have as low as $1 \%$ difference in RMSE values. The algorithm managed to quantify the stiction correctly despite the high similarity. It is recommended to use the first order process model for linear processes since it is faster to be obtained and has less MSE than NARX model. On the other hand, NARX model to be used for highly nonlinear processes.

The algorithm was improved by changing the search algorithm in order to avoid falling in a local minimum. Another improvement was incorporating the search and valve resolutions in the algorithm in order to enable the detection of stiction values with real numbers in industry. Besides that, the algorithm was simplified and its accuracy was improved by using first order process model. Finally, the algorithm's process model dependency was eliminated by applying it on OP-MV data by simulating plant smart instrumentation.

As a conclusion, the five case studies showed that the algorithm is robust since it quantified the stiction correctly regardless of the operating conditions. Therefore, it can be applied on any type of process. The accuracy of the quantification results depends on the following factors:

a) Process model accuracy such as in NARX and FOPTD

b) Number of data samples used in RMSE calculation

c) Search resolution value.

In a nutshell, the research has met its objective since it improved the current algorithm and proved its robustness in several operating conditions. It is recommended as future work to test the algorithm performance under a different stiction model such the one developed by Kanu et al. (2004) against Choudhury's model. 


\section{ACKNOWLEDGEMENT}

The authors would like to thank UTP for the funding and support provided for this work.

\section{REFERENCES}

1. Al-Samarraie, S.A. (2013). "Invariant sets in sliding mode control theory with application to servo actuator system with friction", WSEAS Trans. Syst. Control, 8(2), pp. 33-45.

2. Ana S.R. Brásio, Romanenko Andry and Natércia C.P. Fernandes. (2014). Modeling, detection and quantification, and compensation of stiction in control loops: the state of the art. J. Ind. Eng. Chem. Res., 53, pp. 15020-15040

3. Bedoui, H., Khedher, A., Othman, K.B. (2014). "Fault detection and isolation of fuzzy system with uncertain parameters using the bounded approach", WSEAS Trans. Syst. Control, 9, pp. 269-276.

4. Claudio Scali, Emanuele Matteucci, Daniela Pestonesi, Antonino Zizzo, and Evaldo Bartaloni. (2011). "Experimental characterization and diagnosis of different problems in control valves," XVII Int. Federation of Automatic Control World Congr., pp. 7334-7339.

5. Control Valve Handbook. (2005). 4th ed., Fisher Controls International LLC Marshalltown, IA,. pp. 1-4, 67-74.

6. Dale E. Seborg, Thomas F. Edgar, Duncan A. Mellichamp and Francis J. Doyle III. (2011). "Control system instrumentation" in Process Dynamics and Control, 3rd ed. New Jersey: Wiley, pp. 147-151.

7. Zabiri, H., Maulud, A. and Omar, N. (2009). "NN-based algorithm for control valve stiction quantification," WSEAS Trans. Syst. Control, vol.4, pp. 88-97,.

8. Zabiri, H. and Yudi Samyudia. (2006). "A hybrid formulation and design of model predictive control for systems under actuator saturation and backlash," J. Process Control, vol.16.7, pp. 693-709.

9. Zabiri, H., Ramasamy, M. and Tehv, I.S.Y. (2009). "Quantification analysis for NLPCA-based stiction diagnostic tool," in Int. Conf. on Advanced Computer Control, pp. 468-472.

10. Beall, J. (2010). "Improving control valve performance," Emerson Process Management Corp., Austin, TX, Tech. Rep. 117(10), Aug.

11. Shoukat Choudhury, M.A.A., Maridul Jain and Shah, S.L. (2008). "Stiction definition, modelling, detection and quantification," J. Process Control, vol. 18.3-4, pp. 232-243.

12. Shoukat Choudhury, M.A.A., Thornhill, N.F. and Shah, S.L. (2005). "Modelling valve stiction," J. Control Engineering Practice, vol.13.5, pp. 641-58.

13. M.A.A. Shoukat Choudhury, Sirish L. Shah, Nina F. Thornhill and David S. Shook. "Automatic detection and quantification of stiction in control valves," J. Control Engineering Practice, vol.14, pp. 1395-1412, 2006.

14. Shoukat Choudhury, M.A.A., Shah, S.L. and Thornhill, N.F. (2010). "Diagnosis of poor control-loop performance using higher-order statistics," J. Automatica, vol.40.10, pp. 1719-1728. 
15. Manabu Kanu, Hiroshi Maruta, Hidekazu Kugemoto, and Keiko Shimizu. (2004). "Practical model and detection algorithm for valve stiction," in Int. Federation of Automatic Control Symp. on dynamics and control of process systems, pp. 5-7.

16. Mohieddine Jelali and Biao Huang. (2010). Detection and diagnosis of stiction in control loops: state of the art and advanced methods. [Online]. Available:

http://link.springer.com/chapter/10.10 07/978-1-84882-775-2 2

17. Bacci di Capaci, R., Scali, C., Pestonesi, D. and Bartaloni, E. (2013). "Advanced diagnosis of control loops: Experimentation on pilot plant and validation on industrial scale," Int. Federation of Automatic Control Symp. on dynamics and control of process systems, pp. 589-594.

18. Bacci di Capaci, R. and Scali, C. (2015). "Review on Valve Stiction. Part I: From Modeling to Smart Diagnosis," J. Processes, vol. 3(2), pp. 422-45.

19. Shardt, Y., Zhao, Y., Qi, F., Lee, K., Yu, X., Huang, B. and Shah, S. (2012). "Determining the state of a process control system: Current trends and future challenges," J. Process Control, vol. 90(2), pp. 217-245. 